\title{
Impact of respiratory viruses in the neonatal intensive care unit
}

\author{
Jessica E. Shui ${ }^{1}$ Maria Messina ${ }^{2,3} \cdot$ Alexandra C. Hill-Ricciuti $\mathbb{C}^{2} \cdot$ Philip Maykowski $^{2,4} \cdot$ Tina Leone $^{1} \cdot$ Rakesh Sahni $^{1}$. \\ Joseph R. Isler ${ }^{1} \cdot$ Lisa Saiman ${ }^{2,3}$
}

Received: 30 March 2018 / Revised: 5 July 2018 / Accepted: 30 July 2018 / Published online: 29 August 2018

(c) Springer Nature America, Inc. 2018

\begin{abstract}
Objective To describe the epidemiology and clinical impact of respiratory viruses in a neonatal intensive care unit (NICU). Study design We conducted a retrospective observational study of infants with respiratory viruses detected by multiplex reverse-transcriptase PCR from May 2012 to May 2017. The proportion of symptomatic vs. asymptomatic infants and associated morbidity were assessed. The association of infection prevention and control (IP\&C) strategies and transmission was ascertained.

Results Respiratory viruses were detected in 83 infants representing 86 unique episodes during which infants remained asymptomatic in 15 (17\%). Of the 71 symptomatic episodes, only $45 \%$ were associated with increased respiratory and/or nutritional support. Rhinovirus/enteroviruses were most common (69\%) and involved nine of 12 transmission events. IP\&C strategies including restricting visitors $<12$ years of age and screening exposed infants were associated with decreased transmission rates.

Conclusions NICU patients can be asymptomatic carriers of respiratory viruses. Identification of such infants is important to prevent transmission in the NICU.
\end{abstract}

\section{Introduction}

Respiratory viruses in infants hospitalized in the neonatal intensive care unit (NICU) are associated with a variety of clinical presentations ranging from localized upper respiratory tract infection to disseminated disease to death $[1,2]$. While asymptomatic carriage of respiratory viruses is well characterized in older children and adults [3,4], this entity is not as well described in infants in the NICU. Furthermore, few recent studies have assessed the epidemiology of healthcare-associated respiratory viruses in the NICU population during the era of commercially available

Lisa Saiman

Ls5@cumc.columbia.edu

1 Division of Neonatology, Department of Pediatrics, Columbia University Irving Medical Center, New York, NY, USA

2 Division of Infectious Diseases, Department of Pediatrics, Columbia University Irving Medical Center, New York, NY, USA

3 Department of Infection Prevention and Control, New YorkPresbyterian Hospital, New York, NY, USA

4 Present address: University of Arizona College of MedicinePhoenix, Phoenix, AZ, USA multiplex reverse-transcription polymerase chain reaction (RT-PCR) assays nor assessed the potential strategies to reduce acquisition and transmission.

The objectives of this study were to describe the epidemiology of respiratory viruses in the NICU, as assessed by RT-PCR assays; the clinical impact of respiratory viruses, including the proportion of asymptomatic vs. symptomatic infants; and the infection prevention and control (IP\&C) strategies implemented to reduce transmission of respiratory viruses. Additionally, we explored whether clinically asymptomatic infants had an increased frequency of subclinical oxygen desaturation events before and after a positive RT-PCR for a respiratory virus by using the "Advanced Physiological Monitoring" system, BedMasterEx (Excel Medical, Jupiter, FL), which records and stores all oxygen saturation data from infants' pulse oximeters [5].

\section{Methods}

\section{Study design, site, and subjects}

This was a retrospective observational cohort study of infants hospitalized in our NICU from May 2012 to May 
2017. The NICU is a regional perinatal center, level IV facility that cares for both premature infants and full-term infants with complex congenital cardiac and non-cardiac anomalies. The unit is located on two floors and consists of 58 beds, including shared pods with $2-3$ beds each, a ninebed 'step down' open unit, and four single rooms. There were 5240 admissions during the 5-year study period. Included infants were those with healthcare-associated respiratory viruses detected after the incubation period for the relevant virus as compared to their date of NICU admission. To avoid misclassification of hospital-associated infections attributed to our NICU, we excluded infants who were transferred to our NICU and had respiratory viruses detected within the incubation period of the relevant virus as compared to their date of NICU transfer. This study was approved by the Institutional Review Board of Columbia University Irving Medical Center (CUIMC) with a waiver of informed consent.

\section{Sample collection and viral diagnostic testing}

Nasal turbinate samples were collected, regardless of the mode of respiratory support. Diagnostic testing was performed using a multiplex RT-PCR assay (FilmArray, Biofire, Inc.) that was introduced in our facility in May 2012 without restrictions placed on its use. The assay has been validated on samples from infants in the NICU [6], and the respiratory viral panel (RVP) detects 17 viruses: adenovirus; four coronavirus types (229E, HKU1, NL63, and OC43); human metapneumovirus; rhinovirus/enterovirus (RV/EV); influenza A, AH1, AH3, and B; parainfluenza types $1-4$, and respiratory syncytial virus (RSV), as well as pertussis, Mycoplasma, and Chlamydophila [7]. Only qualitative results are provided; viral load results are unavailable.

\section{Case definitions and clinical outcomes}

Infants were classified as symptomatic or asymptomatic based on the review of the infants' electronic medical records. Symptomatic infants were those with signs of a respiratory infection, e.g., rhinorrhea, fever, cough, and/or those who had escalation of respiratory and/ or nutritional support, as defined below. Asymptomatic infants did not have clinical signs of respiratory infection, nor escalation of respiratory or nutritional support.

Escalation of respiratory or nutritional support was assessed by comparing the maximum support during the 7 days prior vs. the maximum support during the 7 days after detection of a respiratory virus. Escalation of respiratory support was defined as (1) an increase in supplemental oxygen by at least $10 \%$ for at least $12 \mathrm{~h}$ while maintaining the same mode of respiratory support; (2) an increased amount of non-invasive support, e.g., room air to nasal cannula (NC) or NC to continuous positive airways pressure (CPAP) or newly initiated non-invasive positive pressure support; (3) transition from non-invasive to invasive support, e.g., CPAP to synchronized intermittent mechanical ventilation (SIMV); and/or (4) increased invasive support, e.g., increase in mean airway pressure, SIMV to high-rate intermittent mechanical ventilation to high-frequency oscillatory ventilation, and/or to extracorporeal membrane oxygenation (ECMO). Escalation of nutritional support was defined as transitioning from (1) full oral feeds to $\geq 50 \%$ enteral tube feeds or (2) $\geq 50 \%$ enteral feeds to only total parenteral nutrition (TPN).

To further describe the study population, we collected secondary diagnoses that occurred throughout the infants' NICU stay, including bronchopulmonary dysplasia (BPD), necrotizing enterocolitis (NEC), severe retinopathy of prematurity (ROP), severe intraventricular hemorrhage (IVH), and hemodynamically significant patent ductus arteriosus (PDA). BPD was defined as requiring supplemental oxygen $\geq 28$ days, and $\mathrm{BPD}$ severity was categorized based on the amount of respiratory support required at 36 weeks corrected gestational age or discharge, whichever occurred first [8]. NEC was defined as modified Bell's staging criteria IIa or higher $[9,10]$. Severe ROP was defined as stage 3 or higher, based on pediatric ophthalmology exam [11]. Severe IVH was defined as grade 3 or higher on any imaging done before day of life 28 [12]. Hemodynamically significant PDA was defined as lesions requiring medical and/or surgical management.

Transmission was identified if infants, who were both geographically and temporally linked, had the same virus detected. Geographic links were considered as the same pod and/or infants in the neighboring rooms. Temporal links were considered those that occurred within the incubation period of the relevant virus. Transmission patterns were categorized as dyads ( $n=2$ infants), clusters ( $n=3$ infants), or outbreaks ( $n>3$ infants). Transmission events were defined as the number of infants who became infected as a result of transmission, e.g., one transmission event in each dyad and two transmission events in each cluster.

\section{Infection prevention and control strategies}

\section{Infants}

Throughout the study period, RT-PCR testing was performed for symptomatic infants (e.g., fever, cough, and increased secretions). Infants with a respiratory virus detected were placed on contact and droplet isolation. Personnel from the Department of IP\&C interviewed the 
staff to determine if the affected infant had been exposed to ill family members or ill healthcare personnel (HCP). The duration of isolation was considered on a case-bycase basis, but generally, isolation continued for the duration of the relevant viral shedding period or the duration of illness, whichever was greater; two negative RT-PCR tests were required to discontinue isolation. Potentially exposed infants (e.g., those sharing the pod with an affected infant) were monitored for signs and symptoms of respiratory infection and tested if symptoms developed.

Starting in January 2015, in response to an adenovirus outbreak, RT-PCR testing was performed for all potentially exposed asymptomatic infants. Infants were considered exposed if they were geographically near a symptomatic virus-positive index case, e.g., the same pod. Unexposed infants were not screened. Testing was performed at the time of potential exposure and at the end of the relevant incubation period for the virus of the index case. Potentially exposed infants were placed on contact and droplet isolation for the duration of the incubation period. Isolation for exposed infants was discontinued if RT-PCR testing was negative, the infant remained asymptomatic, and no additional geographically related cases were identified. Exposed asymptomatic infants with positive RT-PCR testing were managed as described above for symptomatic infants with positive RT-PCR testing.

\section{Healthcare personnel}

HCP were instructed not to work while sick. Ill HCP were tested by Workforce Health \& Safety and obtained clearance to return to work [13]. Since the 2013-2014 influenza season, HCP in New York State have been required to have an influenza vaccination or wear a surgical mask when in areas where patients are typically present [13].

\section{Visitors}

From May 2012 to November 2015, written educational material explained that ill visitors were prohibited from entering the NICU. Starting in December 2015, visitors younger than 12 years of age were prohibited from visiting the NICU year-round. Large signage was posted at the NICU entry detailing visitor restrictions for age or signs and symptoms of illness, e.g., fever and cough. Formal screening of visitors was not performed at any time during the study.

\section{Data collection}

Data were collected from the electronic medical record and included demographic and clinical characteristics of infants, including the indications for RVP testing, respiratory and nutritional support provided in the 7 days before and after detection of a respiratory diagnosis, as well as primary and secondary diagnoses. All secondary diagnoses were collected throughout the NICU stay. Additionally, we recorded whether the infants were fed with breast milk in the week before RVP testing and the percentage of maternal breast milk comprising the feeds, if applicable, to assess whether this might protect the infants from developing symptoms associated with respiratory viruses. Delayed surgery and delayed discharge associated with detection of a respiratory virus and 30-day crude mortality were also collected.

Data for transmission events were derived from records maintained by the Department of IP\&C that also maintained a list of exposed and tested infants, as well as the indications for RVP testing.

Data from the BedMasterEx system were retrieved from the central network and used to corroborate infants as symptomatic vs. asymptomatic. BedMasterEx system continuously records the high-resolution waveform data sampled at $240 \mathrm{~Hz}$ from the bedside GE monitors [5]. For this study, we extracted oxygen saturation $\left(\mathrm{SpO}_{2}\right)$ data as measured by pulse oximeter collected every minute. A custom MATLAB algorithm was used for removing the artifact, based on how a clinician would interpret the data at the bedside [14]. Waveforms that were inconsistent and irregular, based on comparison of hourly trends of minute heart rate and $\mathrm{SpO}_{2}$, were considered unlikely to translate into accurate and meaningful values for $\mathrm{SpO}_{2}$, and were removed. The remaining $\mathrm{SpO}_{2}$ data retained for analysis ( $\sim 95 \%$ of raw data) were summarized as the percentage of time every $24 \mathrm{~h}$ that an infant's $\mathrm{SpO}_{2}$ was $<85 \%$.

\section{Data analysis}

Demographic and clinical characteristics of symptomatic and asymptomatic infants were summarized with frequencies, means, and medians, and were compared using Chi-square or Fisher's exact test, as appropriate, for categorical variables and two sample $t$ tests and Mann-Whitney $U$ tests for continuous variables. The association of detection of respiratory viruses with changes in respiratory and nutritional support was categorized as unchanged, increased, or decreased support. Breastmilk exposure in the week before a positive RVP was dichotomized as $\geq 50 \%$ enteral feeds being maternal breast milk vs. $<50 \%$ and no maternal breastmilk exposure (i.e., only formula fed or no enteral feeds) vs. any breastmilk exposure.

BedMasterEx data for 7 days before and after positive RVP were available for 40 infants (30 symptomatic and ten asymptomatic infants). The percentage of time these infants had $\mathrm{SpO}_{2}<85 \%$ (considered a hypoxemic state) for each of 
Table 1 Demographic and clinical characteristics of symptomatic and asymptomatic infants $(n=83)$ with respiratory viral detection in a neonatal ICU

\begin{tabular}{|c|c|c|c|c|}
\hline Characteristics & Total $(n=83)$ & $\begin{array}{l}\text { Symptomatic infants } \\
(n=70)\end{array}$ & $\begin{array}{l}\text { Asymptomatic infants } \\
(n=13)\end{array}$ & $p$ value \\
\hline \multicolumn{5}{|l|}{ Demographic } \\
\hline $\begin{array}{l}\text { Birthweight, grams (median, } \\
\text { IQR) }\end{array}$ & $\begin{array}{l}990 \\
{[750-2070]}\end{array}$ & 990 [785-2000] & $940[690-2100]$ & 0.73 \\
\hline $\begin{array}{l}\text { Gestational age, weeks (mean, } \\
\text { SD) }\end{array}$ & $30.4( \pm 5.4)$ & $30.5( \pm 5.6)$ & $29.8( \pm 4.8)$ & 0.74 \\
\hline Male $(n, \%)$ & $43(52)$ & $37(53)$ & $6(46)$ & 0.77 \\
\hline Race $(n, \%)$ & & & & 0.82 \\
\hline White & $40(48)$ & $32(46)$ & $8(62)$ & \\
\hline Black & $17(21)$ & $15(22)$ & $2(15)$ & \\
\hline Asian & $1(1)$ & $1(1)$ & $0(0)$ & \\
\hline Other & $5(6)$ & $5(7)$ & $0(0)$ & \\
\hline Unknown & $20(24)$ & $17(24)$ & $3(23)$ & \\
\hline Hispanic ethnicity $(n, \%)$ & $4(5)$ & $3(4)$ & $1(8)$ & 0.34 \\
\hline Multiple gestation $(n, \%)$ & $18(22)$ & $16(23)$ & $2(15)$ & 0.72 \\
\hline \multicolumn{5}{|l|}{ Clinical $(n, \%)$} \\
\hline \multicolumn{5}{|l|}{ Primary diagnosis } \\
\hline$<37$ weeks gestational age & $52(63)$ & $44(63)$ & $8(62)$ & 1.00 \\
\hline Congenital heart disease ${ }^{a}$ & $10(12)$ & $9(13)$ & $1(8)$ & 1.00 \\
\hline $\begin{array}{l}\text { Non-congenital heart disease } \\
\text { anomaly }\end{array}$ & $12(14)$ & $9(13)$ & $3(23)$ & 0.39 \\
\hline Other $^{\mathrm{b}}$ & $9(11)$ & $8(11)$ & $1(8)$ & 1.00 \\
\hline \multicolumn{5}{|l|}{ Secondary diagnoses } \\
\hline Bronchopulmonary dysplasia & $21(25)$ & $18(26)$ & $3(23)$ & 1.00 \\
\hline Mild & $3(4)$ & $3(4)$ & $0(0)$ & 1.00 \\
\hline Moderate & $0(0)$ & $0(0)$ & $0(0)$ & - \\
\hline Severe & $18(22)$ & $15(21)$ & $3(23)$ & 1.00 \\
\hline Necrotizing enterocolitis & $21(25)$ & $16(23)$ & $5(38)$ & 0.30 \\
\hline Retinopathy of prematurity & $15(18)$ & $12(17)$ & $3(23)$ & 0.70 \\
\hline Patent ductus arteriosus & $23(28)$ & $19(27)$ & $4(31)$ & 0.75 \\
\hline $\begin{array}{l}\text { Intraventricular hemorrhage/ } \\
\text { periventricular leukomalacia }\end{array}$ & $12(14)$ & $11(16)$ & $1(8)$ & 0.68 \\
\hline
\end{tabular}

$S D$ standard deviation, $I Q R$ interquartile range

${ }^{a}$ Congential heart disease included diagnoses of Shone's variant, hypoplastic left heart syndrome, coarctation of the aorta, complex heterotaxia, tetralogy of fallot, and truncus arteriosus

${ }^{b}$ Other diagnoses included: meconium aspiration syndrome, neonatal abstinence syndrome, neonatal diabetes, and hypoxic ischemic encephalopathy the 7 days before and after viral detection were calculated and compared using a mixed model for repeated-measures analysis of variance to account for the missing observations, as well as two sample and paired $t$ tests, as appropriate. Infants with physiologic right to left cardiac shunts were excluded from this analysis due to different expectations for their normal $\mathrm{SpO}_{2}$ range.

The incidence of respiratory virus transmission event rates per 1000 patient-days was also calculated and compared for the time periods before and after the selected interventions were implemented using Poisson regression. All statistical analyses were performed in SAS 9.4 (Cary, NC).

\section{Results}

\section{Study subjects}

From May 2012 to May 2017, 1494 RVPs were sent from the NICU from 727 infants. In all, 83 infants experienced 86 episodes of healthcare-associated respiratory viruses; this represented $1.6 \%$ of the overall NICU admissions. One infant had two viruses detected from the same sample and three infants each had two unique episodes with different viruses detected. Thirteen infants had 15 (17\%) asymptomatic episodes and 70 infants had 71 (83\%) symptomatic episodes of viral detection. One infant had both 
Table 2 Detected viruses and selected clinical outcomes of symptomatic vs. asymptomatic respiratory viral detection episodes $(n=86)$ in the NICU

\begin{tabular}{|c|c|c|c|c|}
\hline Variable & Total & $\begin{array}{l}\text { Symptomatic } \\
\text { episodes }(n=71)\end{array}$ & $\begin{array}{l}\text { Asymptomatic episodes } \\
(n=15)^{\mathrm{a}}\end{array}$ & $p$ value \\
\hline \multicolumn{5}{|l|}{ At viral detection } \\
\hline $\begin{array}{l}\text { Chronological age, days } \\
\text { (median, IQR) }\end{array}$ & $82[58-158]$ & $80.5[54.5-158]$ & $97.5[60-125]$ & 0.32 \\
\hline $\begin{array}{l}\text { Post-menstrual age, weeks } \\
\text { (median, IQR) }\end{array}$ & $\begin{array}{l}44.6 \\
{[38.0-50.4]}\end{array}$ & $44.4[37.9-52.4]$ & $42.9[38.3-49.4]$ & 0.74 \\
\hline \multicolumn{5}{|c|}{ Baseline respiratory support $(n, \%)$} \\
\hline Room air & $47(55)$ & $37(52)$ & $10(67)$ & 0.40 \\
\hline Non-invasive support & $37(43)$ & $33(46)$ & $4(27)$ & 0.16 \\
\hline Invasive support & $2(2)$ & $1(1)$ & $1(7)$ & 0.32 \\
\hline \multicolumn{5}{|l|}{ Pathogens $(n, \%)$} \\
\hline Rhinovirus/enterovirus & $60(69)$ & $49(69)$ & $11(69)$ & 1.00 \\
\hline Coronavirus & $6(7)$ & $5(7)$ & $1(6)$ & 1.00 \\
\hline Adenovirus & $7(8)$ & $5(7)$ & $2(12)$ & 0.60 \\
\hline $\begin{array}{l}\text { Respiratory syncytial } \\
\text { virus }\end{array}$ & $5(6)$ & $4(6)$ & $1(6)$ & 1.00 \\
\hline Parainfluenza & $6(7)$ & $6(8)$ & $0(0)$ & 0.59 \\
\hline Influenza & $2(2)$ & $1(1)$ & $1(6)$ & 0.32 \\
\hline Human metapneumovirus & $1(1)$ & $1(1)$ & $0(0)$ & 1.00 \\
\hline \multicolumn{5}{|c|}{ Additional clinical impact $(n, \%)$} \\
\hline Delayed surgery & $4(5)$ & $4(6)$ & $0(0)$ & 0.31 \\
\hline Delayed discharge & $3(4)$ & $3(5)$ & $0(0)$ & 0.31 \\
\hline Readmitted & $1(1)$ & $1(2)$ & $0(0)$ & 0.75 \\
\hline 30-day crude mortality & $6(7)$ & $5(7)$ & $1(1)$ & 1.00 \\
\hline
\end{tabular}

$S D$ standard deviation, $I Q R$ interquartile range

${ }^{a}$ Includes one episode of co-infection asymptomatic and symptomatic episodes. Thirteen additional infants transferred from other hospitals had a respiratory virus detected within the incubation period of the relevant virus (eight RV/EV, two coronavirus, one RSV, one influenza, and one parainfluenza) and were excluded from further analysis.

The demographic and clinical characteristics of symptomatic and asymptomatic infants with healthcareassociated respiratory viruses were similar (Table 1). There was no difference in the proportion of asymptomatic vs. symptomatic infants who received any maternal breastmilk feeds in the week before a positive RVP (31\% of asymptomatic infants and $34 \%$ of symptomatic, $p=1.00$ ). Additionally, the proportion of asymptomatic vs. symptomatic infants whose feeds comprised of $\geq 50 \%$ maternal breast milk in the week before a positive RVP was similar $(29 \%$ vs. $24 \%, p=0.74)$.

\section{Virology}

Infants with healthcare-associated respiratory viruses had a median chronological age of 82 [IQR 58-158] days at viral detection (Table 2). The distribution of the types of viruses detected was similar in symptomatic and asymptomatic infants (Table 2). The most common viruses were RV/EV $(n=60,71 \%)$, followed by adenovirus $(n=7,8 \%)$, parainfluenza $(n=6,7 \%)$, and coronavirus $(n=6,7 \%)$. The relative proportion of RV/EV detection remained similar over time (quarterly mean $65 \%$, range $50-84 \%, p=0.14$ ). Ten (67\%) of 15 asymptomatic episodes were associated with RV/EV, two with adenovirus (14\%), and one each with coronavirus HK, RSV, and influenza.

\section{Clinical impact associated with respiratory virus detection}

The most common indications for viral diagnostic testing among symptomatic infants were suspected upper respiratory tract infections $(67 \%)$, increased respiratory support (29\%), and/or cough (26\%). Most symptomatic episodes were not managed with increased respiratory support (62\%) and/or nutritional support (79\%). However, 17 (24\%) symptomatic episodes were associated with only escalations of respiratory support, five (7\%) with only escalations of nutritional support, and ten (14\%) with both. The escalations in respiratory support included: (1) three episodes associated with $\geq 10 \%$ increase in $\mathrm{FiO}_{2}$ support while maintaining the same mode of respiratory support; (2) 11 
Fig. 1 Mean daily percent time with $\mathrm{SpO}_{2}<85 \%$ for symptomatic vs. asymptomatic infants in the 7 days before and after the detection of a respiratory virus. Mean percent time ( \pm standard error) of $\mathrm{SpO}_{2}$ $<85 \%$ in the 7 days before and testing for selected symptomatic $(n=30)$ and asymptomatic infants $(n=10)$ as recorded in the BedMasterEx database. Significant differences are indicated by $*$ for $p<0.05$ and $* *$ for $p<0.005 . \mathrm{SpO}_{2}$ oxygen saturation after the respiratory viral panel

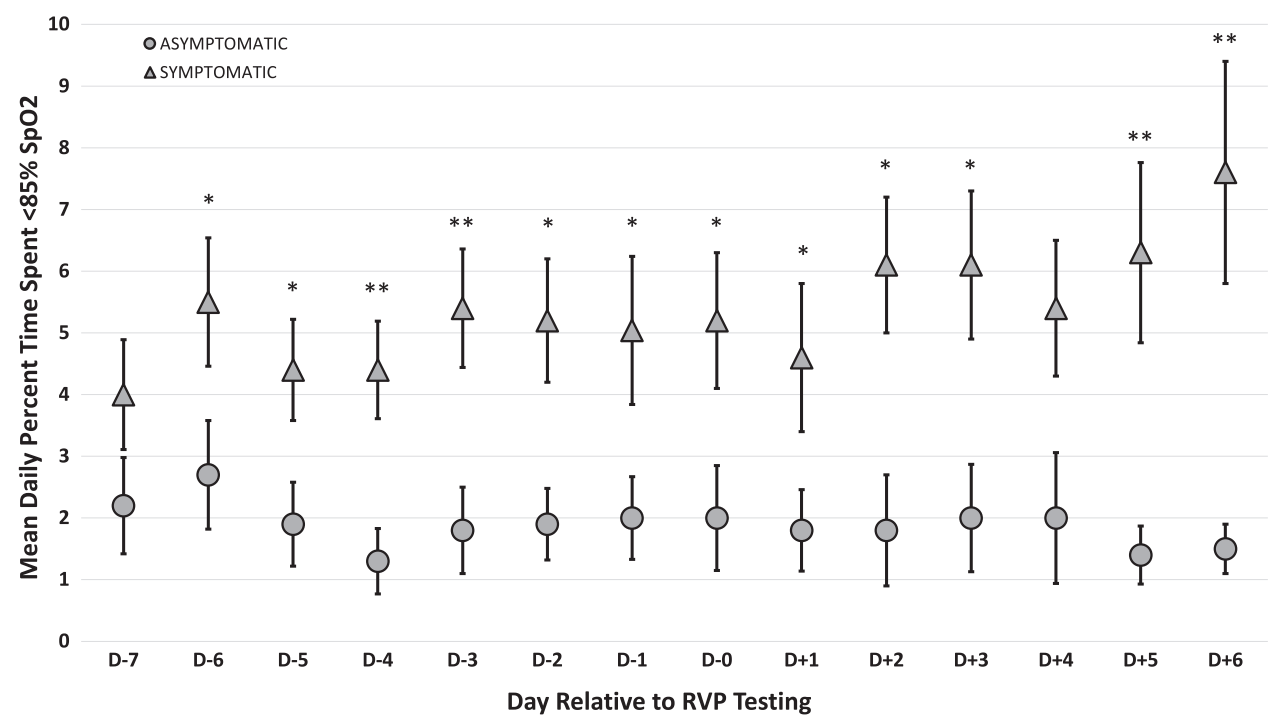

\section{Transmission events}

with increased non-invasive support, most common escalation from room air to CPAP; (3) seven with progression from non-invasive to invasive support, all of which included mechanical ventilation and one with progression to ECMO; and (4) six with increased invasive support. The escalation in nutritional support included: (1) three episodes associated with changing from full oral feeds to $\geq 50 \%$ enteral tube feeds and (2) 12 changing from $\geq 50 \%$ enteral feeds to full TPN support.

The percentage of time the asymptomatic and symptomatic infants had $\mathrm{SpO}_{2}<85 \%$ during the 7 days before and after their positive RVP is shown in Fig. 1. During the 7 days before and after the detection of a respiratory virus, the asymptomatic infants spent significantly less time with $\mathrm{SpO}_{2}<85 \%$, compared with the symptomatic infants (mean daily range $1.3-2.7 \%$ vs. $4.0-7.6 \%$, respectively, $p=0.03$, Fig. 1). The daily percentage of time the asymptomatic infants spent with $\mathrm{SpO}_{2}<85 \%$ did not significantly change throughout the 7 days before and after the viral detection $(p=0.91)$. The daily percentage of time the symptomatic infants spent with $\mathrm{SpO}_{2}<85 \%$ did not significantly change throughout the 7 days before and after the viral detection $(p=0.23)$

Four $(5 \%)$ symptomatic infants had delayed surgery, three $(4 \%)$ had delayed discharge, and one $(1 \%)$ was readmitted one day after NICU discharge with respiratory symptoms due to parainfluenza previously detected in his hospitalized twin. Six infants (7\%) died within 30 days of detection of a respiratory virus; at the time of death, mortality was considered attributable to a respiratory viral infection in two infants (2\%) (one with parainfluenza and one with human metapneumovirus), as ascertained by the NICU team and/or supported by the autopsy findings.
Ten episodes of viral detection were associated with ill family members, and no ill HCP were identified. Six dyad transmission events, four clusters, and two outbreaks were identified (Fig. 2a); nine (75\%) of these 12 transmission events involved RV/EV. Adenovirus ( $n=5$ infants) and $\mathrm{RV} / \mathrm{EV}$ ( $n=7$ infants) each caused an outbreak. Following the introduction of the IP\&C strategy in January 2015 to screen the exposed infants, 82 infants were potentially exposed to 21 case infants, and seven (9\%) of the 82 exposed infants were positive for a respiratory virus. In all, from January 2015 to May 2017, seven (15\%) of 48 episodes of viral detection were identified by screening the exposed infants. Since December 2015, only one transmission event (a dyad of RV/EV in September 2016) occurred; the remaining cases were sporadic. Transmission event incidence per 1000 patient-days is shown in Fig. $2 b$ and decreased significantly from 0.27 per 1000 patient-days before children $<12$ years of age were prohibited from visiting the NICU to 0.03 per 1000 patient-days during the 16 months after children $<12$ years of age were prohibited from visiting the NICU $(p=0.003)$. No transmission events were identified from the 13 transferred infants admitted to the NICU with respiratory viruses.

\section{Discussion}

In this 5-year study, we describe the epidemiology and clinical impact of healthcare-associated respiratory viruses in the NICU population; both sporadic infections and outbreaks occurred. Respiratory viruses were detected in approximately $1.6 \%$ of the overall admissions, but it is somewhat 

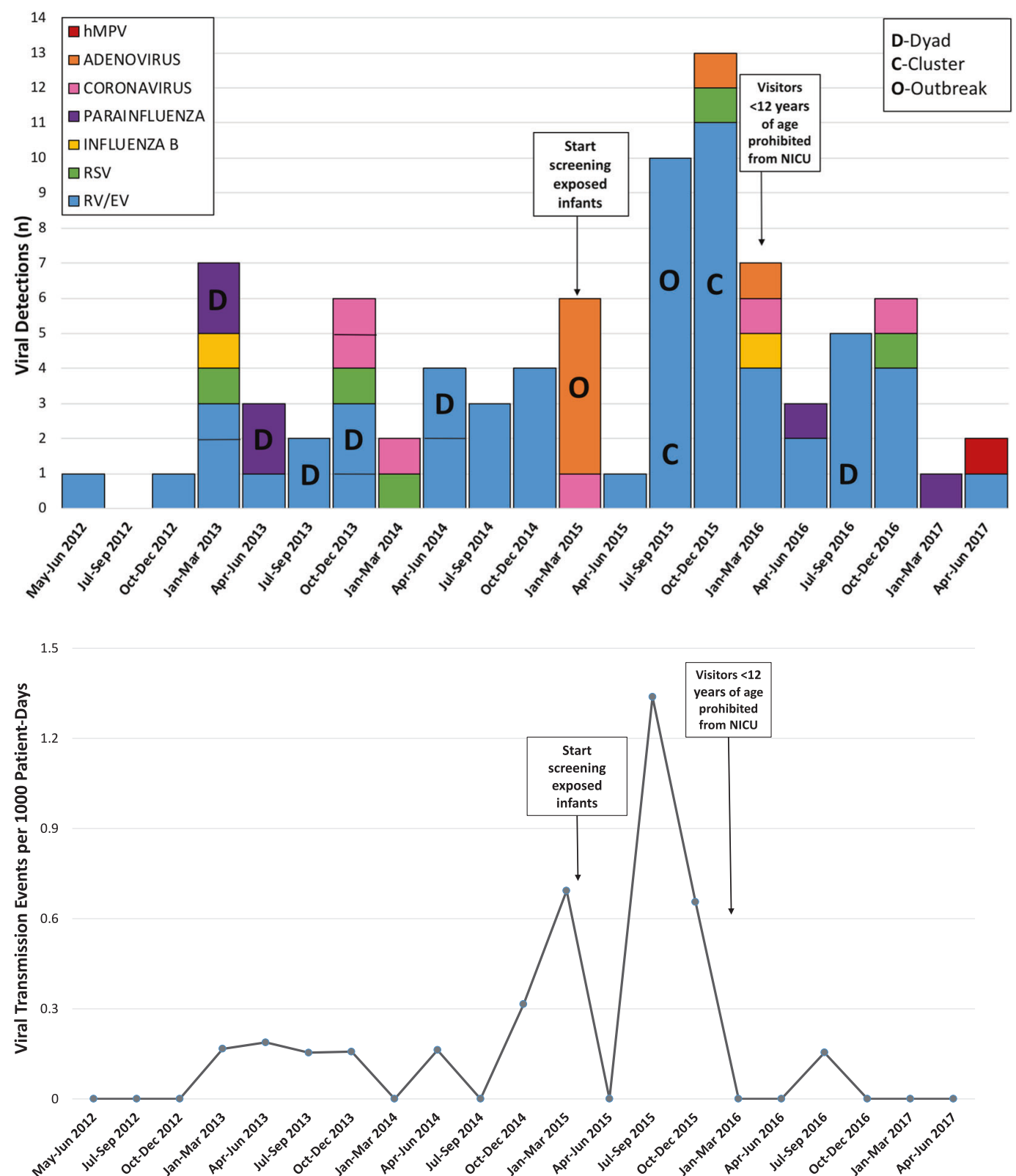

Fig. 2 a Epidemiology and transmission events for respiratory viruses detected from May 2012 to May 2017. a Transmission events were categorized as dyads ( $n=2$ infants), clusters ( $n=3$ infants), or outbreaks ( $n>3$ infants). b Respiratory virus transmission events per 1000 patient-days, May 2012-May 2017. Transmission events were

difficult to find comparable data in previous publications, in which multiplex RT-PCR assays were used during routine clinical care. Others have described clinical surveillance or weekly surveillance for respiratory viruses and demonstrated detection rates ranging from $<1$ to $52 \%$ [15-18]. Different methodologies, patient populations, surveillance strategies, and potentially unidentified outbreaks likely contributed to the wide range of rates reported.

We noted great variability in the symptoms associated with the detection of respiratory viruses, including infants who remained asymptomatic. We also found that most defined as the number of infants who became infected as a result of transmission, e.g., one transmission event in each dyad and two transmission events in each cluster. hMPV human metapneumovirus, $\mathrm{RV} / \mathrm{EV}$ rhinovirus/ enterovirus, RSV respiratory syncytial virus

clinically symptomatic infants did not have increased respiratory and/ or nutritional support. However, some infants did experience substantial morbidity; seven infants progressed from non-invasive to invasive respiratory support, one of whom progressed to ECMO. Others have reported that increases in respiratory support associated with respiratory viruses were associated with an increased length of hospitalization [19-21].

We could not find previous reports of the impact of respiratory viruses on nutritional support, but in the current study, $12(14 \%)$ of 83 infants required escalation to full 
TPN, which likely increased their length of hospitalization. Ten infants had increases in both nutritional and respiratory support. While respiratory viruses do not generally physiologically impact the gastrointestinal tract, concurrent increases in nutritional and respiratory support may have been attributable to feeding intolerance or to providers' concerns for the risk of aspiration and/or hemodynamic instability that could compromise intestinal perfusion. Regardless of the reasons infants received increased nutritional support, these findings provide further evidence of the impact of respiratory viruses in the NICU population.

In the current study, six infants died within 30 days of viral detection, including two infants with attributable mortality. Analysis of a worldwide database found that the mortality rate in NICUs associated with RSV, adenovirus, influenza, and parainfluenza viruses outbreaks was $13.5 \%$, $35.4 \%, 6.9 \%$, and $9.0 \%$, respectively, with an overall virusassociated mortality of $6.1 \%$, although this report included gastrointestinal viruses as well [22, 23]. However, attributable mortality was not ascertained. These findings further support the need to gather additional data on the clinical impact, including mortality, associated with non-congenital healthcare-associated respiratory viruses.

We also found that $17 \%$ of episodes of healthcareassociated respiratory viruses (including two with adenovirus) remained asymptomatic, suggesting that some infants were asymptomatic carriers. Others have also examined asymptomatic infants identified during active surveillance or outbreaks. Caserta et al. [2] performed weekly surveillance for respiratory viruses in 93 hospitalized preterm infants; four had a virus detected including 2 (2\%) who were asymptomatic (rhinovirus and influenza B). Bennet et al. [21] performed weekly surveillance in 50 preterm infants and detected a respiratory virus in 26 infants (52\%); eight (9\%) infants did not have clinical deterioration. During one year of active surveillance, Smit et al. [18] found that $22(7.5 \%)$ of 295 infants without respiratory symptoms had a respiratory virus detected. During an influenza A outbreak, Cunney et al. [24] found that 13 (68\%) of 19 infants remained asymptomatic. Detection of asymptomatic infants has infection control implications; as such infants may transmit a respiratory virus to other infants and/or to HCP.

We were unable to identify a demographic or clinical characteristic associated with asymptomatic infants. However, it is feasible that asymptomatic infants had a lower viral load than symptomatic infants, as has been suggested by others. Jansen et al. [3] found that hospitalized infants less than 1 year of age admitted for an acute respiratory infection had a higher viral load than asymptomatic agematched controls seen in an outpatient clinic (median viral load 63,045 vs. 2856 copies $/ \mathrm{mL}$, respectively, $p=0.02$ ). Similarly, Smit et al. [18] found that infants with a cycle threshold value $(\mathrm{Ct})<35$ were more likely to have respiratory symptoms than those with a $\mathrm{Ct}$ value $>35$. However, we could not explore this factor as viral load measurements are not reported by the Biofire RVP assay.

In a subset of infants, the BedMasterEx's data supported our categorization of asymptomatic and symptomatic infants. During both the 7 days before and after the viral detection, when compared with symptomatic infants, asymptomatic infants spent significantly less time with $\mathrm{SpO}_{2}<85 \%$. However, during the 7 days pre-viral and postviral detection, the daily time spent with $\mathrm{SpO}_{2}<85 \%$ remained similar within the asymptomatic group. Similarly, the daily time spent with $\mathrm{SpO}_{2}<85 \%$ remained similar over time within the symptomatic group. There are several possible explanations for these findings. Symptomatic infants may have had worse baseline lung disease prior to viral detection and thus were at increased risk of becoming symptomatic with the viral infection. Symptomatic infants may have been hypoxemic due to a respiratory viral infection several days prior to being tested. Asymptomatic infants may have had undiagnosed respiratory viral infections previously, and positive tests may have reflected nonviable viruses or viral shedding. Nonetheless, the clinical utility of BedMasterEx data should be further examined in a larger sample of infants to determine if subclinical viral illness can be predicted, thereby prompting earlier testing and potentially reducing transmission.

Our findings suggest that preventing the acquisition and transmission of respiratory viruses in the NICU should be an IP\&C priority. As support for the importance of this priority, during this same time period, 46 central line-associated bloodstream infections occurred in our NICU compared with the 86 episodes of respiratory viral detection described in this study. However, optimal IP\&C strategies for respiratory viruses in the NICU population are unknown and highly variable as demonstrated in a nationwide study conducted in the United Kingdom [25]. Our data suggest that a combination of IP\&C strategies including excluding family members with illnesses, excluding visitors less than 12 years of age year-round, and screening asymptomatic infants in close proximity to an infant with a respiratory virus decreases the transmission event rates, although our sample may have been too small to assess the sustained effect of the interventions over time. Excluding sibling visitors and screeningexposed infants are practices that have been implemented in some NICUs in the U.K [25] and others have shown that restricting sibling visitors reduced RSV [26]. While these interventions were associated with reducing transmission events in our NICU, they did not eliminate respiratory viruses. There are likely multiple 'portals of entry' for respiratory viruses, including unrecognized ill visitors and HCP, adults who are infectious prior to the 
onset of symptoms, and/or asymptomatic adult or infant carriers shedding viruses [4, 27].

\section{Study limitations}

This was a single center observational study which limits its generalizability. It was conducted in a NICU consisting of an open pod design with only four single rooms. Furthermore, our NICU admits full-term infants with congenital anomalies as well as older infants for ROP and BPD management; these admission patterns help to elucidate the relatively older chronologic age of our study population. The overall sample size was small and we could not demonstrate factors associated with being symptomatic vs. asymptomatic, including the distribution of different viruses. The frequency of asymptomatic infants was likely underestimated because the practice for testing exposed infants was not conducted throughout the 5-year study period and we did not test unexposed, asymptomatic infants. It is also possible that unknown confounders or unexamined variables, such as viral load or patterns of care by specific HCP may be responsible for the observed clinical courses of infants in this study. We may have misclassified RV/EV transmission as the Biofire RVP does not distinguish specific virus types nor were the viruses sequenced. We also acknowledge that our strategy to discontinue transmission precautions if an infant has two negative tests does not consider intermittent viral shedding. Finally, we did not measure the impact of culture change in the NICU; following the adenovirus outbreak, staff had increased awareness of the clinical impact of respiratory viruses resulting in increased responsibility and accountability among staff to not work while ill themselves and to ask peers with respiratory symptoms to be evaluated by WH\&S.

\section{Conclusions}

In conclusion, reducing the incidence of healthcareassociated respiratory viruses in the NICU population is an important patient safety goal. Infants in the NICU with respiratory viruses can exhibit a wide range of symptoms including being asymptomatic, as demonstrated by both clinical and physiological data. Remarkably, we found that most symptomatic infants did not receive escalation of respiratory or nutritional support. While the evidence supporting effective infection prevention and control measures is still evolving, we found that screening asymptomatic infants after exposure and prohibiting visitors less than 12 years of age from visiting the NICU were associated with a reduction in incidence and transmission events of respiratory viruses. However, multicenter investigations are needed to assess the generalizability of our findings to identify factors associated with asymptomatic infants and to determine the most cost-effective IP\&C strategies to reduce respiratory viruses in the NICU.

Acknowledgements We would like to acknowledge Dr. Susan Whittier, Director of the Clinical Microbiology Laboratory at CUIMC, for her generosity of time and expertize.

\section{Compliance with ethical standards}

Conflict of interest The authors declare that they have no conflict of interest.

\section{References}

1. Ronchi A, Doern C, Brock E, Pugni L, Sánchez PJ. Neonatal adenoviral infection: a seventeen year experience and review of the literature. J Pediatr. 2014;164:529-35.

2. Caserta MT, Yang H, Gill SR, Holden-Wiltse J, Pryhuber G. Viral respiratory infections in preterm infants during and after hospitalization. J Pediatr. 2017;182:53-8.

3. Jansen RR, Wieringa J, Koekkoek SM, Visser CE, Pakrt D, Molenkamp R, et al. Frequent detection of respiratory viruses without symptoms: toward defining clinically relevant cutoff values. J Clin Microbiol. 2011;49:2631-6.

4. Hassoun A, Huff MD, Weisman D, Chahal K, Asis E, Stalons D, et al. Seasonal variation of respiratory pathogen colonization in asymptomatic health care professionals: a single-center, crosssectional, 2-season observational study. Am J Infect Control. 2015;43:865-70.

5. Excel Medical BedmasterEx. http://excel-medical.com/products/ bedmaster-ex/. Accessed 5 Feb 2018.

6. Piralla A, Lunghi G, Percivalle E, Vigano C, Nasta T, Pugni L, et al. FilmArray ${ }^{\circledR}$ respiratory panel performance in respiratory samples from neonatal care units. Diagn Microbiol Infect Dis. 2014;79:183-6.

7. Poritz MA, Blaschke AJ, Byington CL, Meyers L, Nilsson K, Jones DE, et al. FilmArray, an automated nested multiplex PCR system for multi-pathogen detection: development and application to respiratory tract infection. PLoS ONE. 2011;6: e26047.

8. Jobe AH, Bancalari E. Bronchopulmonary dysplasia. Am J Respir Crit Care Med. 2001;163:1723-9.

9. Walsh MC, Kliegman RM. Necrotizing enterocolitis: treatment based on staging criteria. Pediatr Clin North Am. 1986;33:179-201.

10. Bell MJ, Ternberg JL, Feigin RD, Keating JP, Marshall R, Barton L, et al. Neonatal necrotizing enterocolitis. Therapeutic decisions based upon clinical staging. Ann Surg. 1978;187:1-7.

11. International Committee for the Classification of Retinopathy of Prematurity. The international classification of retinopathy of prematurity revisited. Arch Ophthalmol. 2005;123:991-9.

12. Papile LA, Burstein J, Burstein R, Koffler H. Incidence and evolution of subendymal and intraventricular hemorrhage: a study of infants with birthweights less than 1500 grams. J Pediatr. 1978;92:529-34.

13. Batabyal RA, Zhou JJ, Howell JD, Alba L, Lee HH, Furuya EY, et al. Impact of New York state influenza mandate on influenzalike illness, acute respiratory illness, and confirmed influenza in healthcare personnel. Infect Control Hosp Epidemiol. 2017;38:1361-3. 
14. Isler JR, Grieve PG, Weindler M, Bateman DA, Garland M, Stark RI et al. Oxygen saturation profiles in extremely low birth weight (ELBW) infants with and without supplemental oxygen. ESPR Annual Meeting 2015:263.

15. Ronchi A, Michelow IC, Chapin KC, Bliss JM, Pugni L, Mosca F, et al. Viral respiratory tract infections in the neonatal intensive care unit: the virion-i study. J Pediatr. 2014;165:690-6.

16. Verboon-Maciolek MA, Krediet TG, Gerards LJ, Fleer A, Van Loon TM. Clinical and epidemiologic characteristics of viral infections in a neonatal intensive care unit during a 12-year period. Pediatr Infect Dis J. 2005;24:901-4.

17. Kidszun A, Hansmann A, Winter J, Grondahl B, Knuf M, Weise $\mathrm{K}$, et al. Detection of respiratory viral infections in neonates treated for suspicion of nosocomial bacterial sepsis: a feasibility study. Pediatr Infect Dis J. 2014;33:102-4.

18. Smit PM, Pronk SM, Kaandorp JC, Weijer O, Lauw FN, Smits $\mathrm{PH}$, et al. RT-PCR detection of respiratory pathogens in newborn children admitted to a neonatal medium care unit. Pediatr Res. 2013;73:355-61.

19. Steiner M, Strassl R, Straub J, Böhm J, Popow-Kraupp T, Berger A. Nosocomial rhinovirus infection in preterm infants. Pediatr Infect Dis J. 2012;31:1302-4.

20. Delgado-Corcoran C, Witte MK, Ampofo K, Castillo R, Bodily S, Bratton SL. The impact of human rhinovirus infection in pediatric patients undergoing heart surgery. Pediatr Cardiol. 2014;5:387-94.

21. Bennett NJ, Tabarani CM, Bartholomona NM, Wang D, Huang D, Riddell SW, et al. Incidence and implications of unrecognized viral respiratory tract infections in premature infants during their birth hospitalization: a prospective surveillance study in two neonatal intensive care units. J Pediatr. 2012;161:814-8.

22. Civardi E, Tzialla C, Baldanti F, Strocchio L, Manzoni P, Stronati M. Viral outbreaks in neonatal intensive care units: what we do not know. Am J Infect Control. 2013;41:854-6.

23. Tzialla C, Civardi E, Borghesi A, Sarasini A, Baldanti F, Stronati M. Emerging viral infections in neonatal intensive care unit. J Matern Neonatal Med. 2011;24:156-8.

24. Cunney RJ, Bialachowski A, Thornley D, Smaill FM, Pennie RA. An outbreak of influenza A in a neonatal intensive care unit. Infect Control Hosp Epidemiol. 2000;21:449-54.

25. Tan S, Clarkson M, Sharkey D. Variation in visiting and isolation policies in neonatal units. Pediatr Infect Dis J. 2017;37:1.

26. Peluso AM, Harnish BA, Miller NS, Cooper ER, Fujii AM. Effect of young sibling visitation on respiratory syncytial virus activity in a NICU. J Perinatol. 2015;35:627-30.

27. Homaira N, Sheils J, Stelzer-Braid S, Lui K, Oie JL, Snelling T, et al. Respiratory sncytial virus is present in the neonatal intensive care unit. J Med Virol. 2016;88:196-201. 